\title{
La dimensión económica de la pobreza y precariedad urbana en las ciudades latinoamericanas. Implicaciones para las políticas del hábitat
}

\author{
LUCY WINCHESTER* \\ ** Instituto Latinoamericano de Planificación Económica Social (ILPES), CEPAL, Naciones Unidas
}

\begin{abstract}
In Latin American and Caribbean cities, the urban poor are subject to diverse and heterogeneous living conditions that directly and indirectly reflect their economic vulnerability. This article seeks to deepen our understanding of the economic dimension of urban poverty and precarious living conditions in the lives of the urban poor, and to investigate their implications for public policy design. The work relies on the sustainable development framework and the policy discussions regarding capacity building and the creation of opportunities for the poor. Our working hypothesis is that an awareness of the economic vulnerability of the urban poor has not been fully integrated into urban policies, limiting their contribution to improving the poor's access to stable incomes and employment.
\end{abstract}

KEYWORDS: urban poverty, precarious living conditions, economic vulnerability, public policy.

RESUMEN En las ciudades de América Latina y el Caribe, los pobres urbanos viven una heterogeneidad de condiciones y carencias que expresan directamente e indirectamente su vulnerabilidad económica. Este artículo busca profundizar nuestra comprensión de la dimensión económica de la pobreza y precariedad urbana en las vidas de los pobres urbanos, y averiguar sobre sus implicancias en el diseño de estrategias de política. El trabajo se basa en los enfoques conceptuales del desarrollo sostenible, y de la generación de capacidades y oportunidades en las personas. Se parte de una hipótesis de trabajo de que la vulnerabilidad económica en los sectores de pobreza urbana no ha sido plenamente integrada en las políticas del hábitat, limitando la contribución de éstas a la mejora en el acceso de los pobres urbanos al empleo e ingresos estables.

PALABRAS CLAVE: pobreza urbana, precariedad, vulnerabilidad económica, política pública.

\footnotetext{
* Los contenidos de este artículo fueron desarrollados a partir del conjunto de estudios y actividades realizados en el marco del proyecto "Pobreza urbana: estrategia orientada a la acción para los gobiernos e instituciones municipales en América Latina y el Caribe”, llevado a cabo por CEPAL entre 2003 y 2005. Las opiniones expresadas en el trabajo son de estricta responsabilidad de su autora, y no representan aquellos de la organización CEPAL.
}

Recibido el 3 de noviembre de 2008, aprobado el 14 de noviembre de 2008.

Correspondencia: Lucy Winchester, Casilla 179-D, Santiago, Chile. E-mail: lucy.winchester@cepal.org. 


\section{Introducción}

En las ciudades de América Latina y el Caribe, los pobres urbanos viven una heterogeneidad de condiciones y carencias que expresan directamente e indirectamente su vulnerabilidad económica. En nuestra región, la pobreza urbana adopta la forma de bajos ingresos vinculados a empleos precarios, falta de capital educativo y activos patrimoniales, y refleja inequidades por razones de género ${ }^{1}$. La precariedad urbana -entendida como la proporción de los hogares que no tienen cubiertas sus necesidades habitacionales, tales como la materialidad de la vivienda, acceso a servicios (agua y saneamiento) y la tenencia- afecta tanto a los hogares pobres, como también, aunque en menor grado, a los hogares que se encuentran sobre la línea de pobreza por ingresos.

Este artículo busca profundizar nuestra comprensión de la dimensión económica de la pobreza y precariedad urbana en las vidas de los pobres urbanos, y averiguar sobre sus implicancias en el diseño de estrategias de política. Se parte de la hipótesis de trabajo de que la vulnerabilidad económica en los sectores de pobreza urbana no ha sido plenamente integrada en las políticas del hábitat, limitando la contribución de éstas a la mejora en el acceso de los pobres urbanos al empleo e ingresos estables. El trabajo intenta girar la mirada desde los indicadores agregados sobre la situación de pobreza urbana y precariedad en la región, para incorporar al hogar pobre, y ver cómo la dimensión económica influye sobre la vida de los sectores de pobreza, y sobre todo, indagar sobre qué significado esta dinámica tiene para las políticas públicas del hábitat.

El presente trabajo, por tanto, pretende (i) conceptualizar la pobreza y precariedad urbana en su dimensión económica; (ii) entender las expresiones de la dimensión económica de la pobreza y precariedad urbana y su dinámica en la urbe; (iii) entender las estrategias públicas actuales desde el campo del hábitat y la intervención urbano-territorial para atender esta situación y (iv) formular conclusiones y recomendaciones de política. Así, el trabajo busca atender un vacío en el marco de desarrollo que orienta la política pública del hábitat y de las intervenciones urbano-territoriales, para mitigar los impactos de la vulnerabilidad económica de los sectores de pobreza urbana y mejorar su acceso al empleo e ingresos estables.

\section{La pobreza y precariedad urbana: aportes conceptuales}

La manera en que se entiende la pobreza tiene consecuencias en los modelos de intervención que se proponen e implementan. La pobreza es la condición de privación y ausencia de oportunidades de las familias y comunidades a los bienes y servicios que la sociedad, en un momento dado del tiempo, entiende como adecuados para el desarrollo integral del ser humano. Involucra aspectos materiales y no materiales. La pobreza es heterogénea, compleja y dinámica, sus causas son múltiples y operan en el nivel micro, meso y macro social. Se asocia la pobreza con las carencias, pero los pobres tienen recursos y administran sus oportunidades.

\footnotetext{
$1 \quad$ Los hogares dirigidos por mujeres, además de ser excesivamente numerosos entre los pobres, tienden a ser más vulnerables en lo económico y social debido al menor número de trabajadores en cada uno de ellos, lo que aumenta la posibilidad de que caigan más allá de la línea de pobreza en épocas de crisis.
} 
Entre ellas se cuentan recursos materiales que han logrado acumular en el tiempo y recursos sociales y culturales. A continuación, se revisan los enfoques conceptuales principales en torno a la problemática de la pobreza y precariedad urbana.

Los enfoques conceptuales tradicionales de carencias y necesidades básicas insatisfechas, son aquellos que ponen atención sobre la dimensión de las carencias o déficits que presentan grupos de población en indicadores o estándares entendidos como básicos de acuerdo a convenciones internacionales. La pobreza y la desigualdad se miden en términos de ingreso per cápita del hogar. De esta manera se distinguen los pobres indigentes de los pobres no indigentes y los no pobres. Las variaciones de los índices de pobreza e indigencia dependen de la situación del mercado de trabajo y de las oportunidades para generar ingresos por cuenta propia. La perspectiva de necesidades habitacionales insatisfechas - materialidad de la vivienda, acceso a agua potable y saneamiento, tenencia-, actualmente orienta la política habitacional en América Latina y el Caribe, donde proporciona objetivos agregados sobre los déficits cuantitativos y cualitativos, al marco de desarrollo de la política.

Estos enfoques tradicionales no consideran factores psicosociales y formas culturales que tienen las personas para enfrentar y asumir sus propios procesos de desarrollo. Un enfoque centrado en el capital humano reconoce explícitamente que los pobres tienen un capital humano inicial que proporciona posibilidades de superar su condición socioeconómica desmejorada. Desde este segundo enfoque surgen políticas y programas de desarrollo de capital humano. No obstante, desde la perspectiva de desarrollo humano se les critica la falta de una perspectiva integral del desarrollo. En este sentido, se incorpora al enfoque de desarrollo humano del Programa Naciones Unidas para el Desarrollo (PNUD), las teorías de capital social, el enfoque de creación de capacidades y las que se refieren a la política social como garante de los derechos de ciudadanía.

El enfoque de desarrollo humano del PNUD (1997) contribuye a distinguir desarrollo económico y desarrollo social, señalando que, aun cuando hay crecimiento económico, ello en nada garantiza que se vea también comprometido el desarrollo humano. Este enfoque pone énfasis en crear un entorno propicio en el cual las personas pueden realizar sus posibilidades, y vivir plenamente ${ }^{2}$.

La temática del capital social refiere a las relaciones de reciprocidad y confianza entre las personas y grupos, la densidad y características del tejido social, y la naturaleza de los lazos que unen con otros. Lo definitorio del capital social es que es relacional, y tiene que ver con las conexiones y redes entre las personas y grupos inmersos en la estructura social. Derivan del fortalecimiento del capital social políticas de fortalecimiento real de la asociatividad, la participación ciudadana y la colaboración recíproca en iniciativas de interés común (Arriagada, Miranda y Pavez, 2004).

2 Este enfoque tiene origen en el trabajo de Amartya Sen. 
El enfoque de la generación de capacidades entiende que la superación de la pobreza pasa por las personas, adquiriendo importancia temas tales como expansión de competencias personales y comunicacionales, visualización de nuevas posibilidades de crecimiento personal, y autoestima. Plantea que es necesario afectar la trama de interacciones o vínculos que interrelacionan las personas entre sí. Intervenir para modificar estas relaciones requiere trabajar y modificar actitudes, disposiciones y esquemas mentales de los sectores pobres y los "no pobres".

El enfoque de los derechos y la ciudadanía (CEPAL, 2000) plantea que los Estados deben asumir responsabilidades en la garantía de derechos de todos los ciudadanos. Estos derechos comprenden, además de los civiles y políticos, los derechos sociales que constituyen la ciudadanía social. Deriva de esta perspectiva la preocupación por establecer mínimos sociales que son irrenunciables desde el punto de vista de la política pública. Estos mínimos se determinan en función de disminuir las brechas de determinados grupos de población en relación al conjunto de la sociedad.

Para analizar la pobreza desde una perspectiva de género hay que hacer visibles diversas relaciones de poder, como las ligadas a las exclusiones, desigualdades y discriminaciones de género en el mercado laboral, el reparto desigual del trabajo no remunerado, el ejercicio de la violencia física y simbólica en contra de la mujer y el diferente uso del tiempo de hombres y mujeres (Arriagada, 2005).

La pobreza es un fenómeno complejo y multidimensional, que abarca privaciones en muchos aspectos del bienestar individual y colectivo. Para avanzar hacia la superación de la pobreza hay que comprender que la pobreza y el desarrollo se relacionan tanto con condiciones esenciales (intangibles, es decir marcadas en actitudes, esquemas mentales, confianza en sí mismo y frente a otras personas y grupos) como con condiciones necesarias pero no suficientes (nivel de ingresos, empleo, necesidades básicas, aspectos materiales), estando ambas en una relación de interdependencia.

La pobreza urbana ha sido definida mediante los mismos conceptos y atributos utilizados para definir la pobreza en general, a los cuales se han agregado peculiaridades propias de lo urbano. De acuerdo a la Conferencia de Recife, la pobreza urbana es también una condición multidimensional, sujeta a influencias culturales, sociales y locales, entendida subjetivamente y experimentada de manera diferente por los pobres según su sexo, edad, origen étnico y aptitudes (FIPU, 1996). Estudios identifican atributos específicos de la pobreza que sólo es posible encontrar en las ciudades, los que introducen grados de variación que, sin alterar la comprensión general del problema, transforman la pobreza urbana en un tema específico. Estos incluyen:

- una vulnerabilidad resultante de la integración de los sectores urbanos de pobreza a la economía de mercado (cash economy);

- la heterogeneidad socio-económica dentro de lo urbano y respecto a la interacción con los procesos y actividades económicos;

- la segregación socio-espacial;

- la fragmentación social, la inestabilidad de redes y la violencia; 
- los riesgos ambientales y de salud;

- el acceso deficiente a bienes y servicios en las dimensiones de calidad y seguridad.

\section{Los pobres urbanos y su relación con la economía de mercado}

Cabe destacar una condición específica de los hogares pobres urbanos que hace que su vida difiera a la de sus contrapartes rurales, y que marca fuertemente la dinámica de reproducción y producción de estos sectores en el área urbana.

Los hogares pobres urbanos se comparten muchas situaciones con sus contrapartes rurales. Sin embargo, se asocian matices específicos a tanto áreas urbanas como áreas rurales. Por ejemplo, los retornos (en niveles de ingresos) a la educación son mayores en áreas urbanas que rurales. Y con respecto a los mercados laborales, mientras el autoempleo caracteriza la inserción laboral en áreas rurales, es la situación del subempleo la que es característica de las zonas urbanas.

El Banco Mundial (2004) cita algunos análisis de la pobreza en América Latina (Wodon et al, 2001) que determinan que muchos factores explicativos de la pobreza son los mismos entre áreas urbanas y áreas rurales: entre esos factores se pueden enumerar, tamaño de hogar (más grande el hogar, mayor probabilidad de pobreza), jefe de hogar joven o mujer, bajos niveles de educación y situaciones de desempleo (personas activamente buscando trabajo) del jefe/a de hogar y su pareja.

Tal vez la característica más significativa de la dimensión económica en la vida de los pobres urbanos es que éstos se enfrentan fuertemente con la economía del mercado, más que los pobres rurales (Banco Mundial, 2004). Vivir en la ciudad significa tener que generar ingresos para sobrevivir, exigiéndoles a los pobres urbanos integrarse, de una u otra forma, a los mercados laborales. Ellos deben vender su trabajo a cambio de ingresos monetarios. En áreas rurales, aún existen modalidades de generar sustento (muchas veces de subsistencia) donde se transan especies y la fuerza de trabajo (en forma individual y colectiva), además de la producción agrícola directa.

Los ingresos provenientes del trabajo remunerado representan un $80 \%$ del total de los ingresos monetarios de los pobres urbanos latinoamericanos (Banco Mundial, 2004). La posibilidad de vender su trabajo y el nivel de remuneración son factores importantes en sus estrategias de generar sustento. La integración a la economía del mercado significa que los pobres urbanos son mucho más vulnerables a shocks económicos que sus contrapartes rurales, situación que realza la importancia de los mecanismos de sobrevivencia y de adaptación de los hogares, y la comprensión de estos para el desarrollo de estrategias de intervención.

Los shocks macroeconómicos se transmiten a los hogares pobres urbanos a través del mercado laboral, siendo el desempleo en América Latina particularmente urbano (15\% promedio en áreas urbanas versus 3\% en zonas rurales). Es importante destacar que las áreas urbanas de la región se caracterizan por una heterogeneidad de situaciones en el nivel de ocupación y de desempleo de los sectores de escasos recursos, por país, y por género (Banco Mundial, 2004). 
Esta situación, combinada con la heterogeneidad del territorio urbano y sus procesos y actividades económicas, hace difícil y complejo anticiparse a los efectos de los shocks externos en los distintos sectores sociales.

El Cuadro 1 demuestra la interdependencia tanto entre niveles como estabilidad de ingresos de los pobres urbanos, y sus situaciones de carencias y su capacidad de superar dichas circunstancias.

Cuadro 1. Dimensión económica de la pobreza y precariedad urbana. Vínculos entre distintas carencias $\mathrm{y}$ condiciones y niveles reales de ingresos.

\begin{tabular}{|c|c|c|}
\hline Condiciones o carencias & $\begin{array}{l}\text { Relación con niveles de ingresos (hogar } \\
\text { e individual) }\end{array}$ & $\begin{array}{c}\text { Efectos multiplicadores con otros } \\
\text { aspectos de la pobreza urbana }\end{array}$ \\
\hline $\begin{array}{l}\text { Cartera de activos (tangible y } \\
\text { no tangible) inadecuada e ines- } \\
\text { table para individuos, hogares } \\
\text { y comunidades }\end{array}$ & $\begin{array}{l}\text { Relación directa con ingresos: capacidad limitada } \\
\text { para aumentar cartera de activos; se venden activos } \\
\text { para adaptarse a ingresos insuficientes. Acceso } \\
\text { a activos tangibles vía crédito afectan ingresos; } \\
\text { aunque familias pueden acceder activos (vivienda, } \\
\text { tenencia, infraestructura) vía construcción social } \\
\text { del hábitat y negociación con autoridades. } \\
\end{array}$ & $\begin{array}{l}\text { Falta de activos limita acceso a crédito (aval) } \\
\text { para actividades productivas, vivienda e } \\
\text { infraestructura. }\end{array}$ \\
\hline $\begin{array}{l}\text { Vivienda de mala calidad, in- } \\
\text { segura, en áreas de riesgo, y } \\
\text { hacinada }\end{array}$ & $\begin{array}{l}\text { Relación directa con ingreso ya que calidad de } \\
\text { vivienda y seguridad de tenencia se relacionan } \\
\text { con capacidad de pago; estrategia de autocons- } \\
\text { trucción, calidad de construcción marginal e } \\
\text { inseguridad de la tenencia en la espera que se } \\
\text { legalice situación dominial. }\end{array}$ & $\begin{array}{l}\text { Vivienda insegura y de calidad inadecuada } \\
\text { exhibe riesgos de pérdida de activo; mayores } \\
\text { costos de manutención por uso de materiales } \\
\text { ligeros. Valor limitado de vivienda como } \\
\text { activo que genera ingresos (arriendo de } \\
\text { piezas, taller). }\end{array}$ \\
\hline $\begin{array}{l}\text { Provisión inadecuada de infra- } \\
\text { estructura colectiva (a) }\end{array}$ & $\begin{array}{l}\text { Alguna relación con ingresos ya que provisión } \\
\text { depende de capacidad de pago; sin embargo, } \\
\text { influye mucho disposición de gobierno de } \\
\text { provisión del bien y el nivel de eficiencia de ésta } \\
\text { (costo y calidad de provisión) }\end{array}$ & $\begin{array}{l}\text { Costos más altos. Riesgo de salud puede influir } \\
\text { sobres mayores costos en salud y perdida de } \\
\text { días de trabajo. }\end{array}$ \\
\hline $\begin{array}{l}\text { Provisión inadecuada de servi- } \\
\text { cios básicos (b) }\end{array}$ & $\begin{array}{l}\text { Provisión de servicios relacionados más a la } \\
\text { capacidad de provisión de gobierno que a nivel } \\
\text { de ingresos del hogar. }\end{array}$ & $\begin{array}{l}\text { Falta de educación puede influir sobre ingresos } \\
\text { a futuro; similar para temas de salud. Costos de } \\
\text { transporte representa un porcentaje significativo } \\
\text { de los gastos de los hogares pobres. }\end{array}$ \\
\hline $\begin{array}{l}\text { Redes de protección social inse- } \\
\text { guras o limitadas; inseguridad } \\
\text { urbana y vulnerabilidad urbana; } \\
\text { falta de poder en sistemas de } \\
\text { control social, de representación } \\
\text { y de decisión política }\end{array}$ & $\begin{array}{l}\text { Provisión relacionada a sistema de governance; } \\
\text { Estado de derecho; etc., que deben estar dispo- } \\
\text { nibles a todos los ciudadanos independiente de } \\
\text { su nivel de ingresos. }\end{array}$ & \\
\hline
\end{tabular}

Fuente: Mitlin y Satterthwaite, 2004.

Nota: (a) agua potable, saneamiento, drenaje, calles, espacio público; (b) cuidado infantil/capacitación vocacional/ escuelas, salud, servicios de emergencia, transporte público, comunicaciones y seguridad.

\section{Panorama regional sobre la pobreza y precariedad urbana}

En cuanto a la magnitud del problema de la pobreza en América Latina, las más recientes estimaciones de la CEPAL (2007) indican que en el año 2006, el 37\% de la población de la región se hallaba en situación de pobreza (31,1\% para las áreas urbanas). Un $13 \%$ de esta 
gente es extremadamente pobre o indigente (8,6\% para las áreas urbanas) ${ }^{3}$. Esto significa que casi 200 millones de personas en la región son pobres (127,6 millones en áreas urbanas) y un poco más de 70 millones de personas son extremadamente pobres (35,2 millones en áreas urbanas). Las tasas de pobreza e indigencia indicadas significan descensos respecto a sus niveles de 1990, tanto a nivel nacional como urbano.

El progreso es alentador, pero no alcanza para cumplir con los Objetivos de Desarrollo del Milenio, ya que transcurrió más del 50 por ciento del período que se estableció para alcanzar las metas correspondientes a la pobreza. El crecimiento del ingreso promedio en la mayoría de los países de la región ha sido insuficiente para superar los problemas de la pobreza. Las bajas tasas de crecimiento económico han tenido efectos negativos en el empleo y en la creación de nuevos puestos de trabajo, en especial en las áreas urbanas. Más del $40 \%$ de la población urbana en América Latina está empleada en sectores de baja productividad en los mercados de trabajo (CEPAL, 2007). Ésta es una característica relativamente constante de los mercados laborales urbanos de la región durante los últimos 15 años. Asimismo, los índices nacionales de desigualdad continúan siendo elevados o han sufrido un deterioro absoluto. Los análisis de la CEPAL demuestran que los factores vinculados al bienestar económico tienen efecto tanto sobre la inequidad como sobre la pobreza.

El problema más generalizado que se observa entre los hogares pobres es la falta de acceso al saneamiento, seguido por la incapacidad de asegurar la tenencia de la vivienda y acceder a redes de agua potable. El siguiente factor en importancia es el problema de las viviendas de mala construcción. Finalmente, el hacinamiento continúa siendo una característica predominante de los hogares urbanos pobres. El desafío principal de los sectores urbanos de pobreza, respecto al tema de los servicios urbanos (agua y saneamiento) es la calidad y el acceso efectivo. En general, existen grandes variaciones entre pobres urbanos en el acceso a los servicios (de buena o deficiente calidad), siendo en gran medida la edad del asentamiento el factor determinante (por ejemplo, los nuevos asentamientos irregulares peri-urbanos habitualmente carecen de servicios) (Banco Mundial, 2004).

Existen dos mercados para lograr el acceso al suelo en las urbes: los sectores más prósperos cuentan con un acceso legal seguro a través del mercado formal, en tanto que los sectores de bajos ingresos aplican estrategias de supervivencia recurriendo al mercado informal u ocupando tierras de alto riesgo urbano-ambiental. En la actualidad, según las distintas ciudades/ áreas metropolitanas latinoamericanas, una cantidad más o menos importante de población (en algunos de ellas puede llegar a cerca de 50\%) viven en la informalidad desde el punto de vista dominial, es decir que está ocupando suelo público, y en menor medida, privado, de manera ilegal.

\footnotetext{
3 El enfoque utilizado por la CEPAL para estimar la pobreza consiste en clasificar como "pobre" a una persona cuando el ingreso por habitante de su hogar es inferior al valor de la "línea de pobreza" o monto mínimo necesario para satisfacer sus necesidades esenciales. Las líneas de pobreza, expresadas en la moneda de cada país, se determinan a partir del valor de una canasta de bienes y servicios, empleando el método del "costo de necesidades básicas" (CEPAL, 2007).
} 
El mayor obstáculo para que la población consiga la seguridad de la tenencia se basa en el derecho de propiedad del suelo a través del mercado. El suelo se convierte en una mercancía más que se compra y vende en particulares mercados -legal e ilegal-; la población que no puede acceder al mercado legal, infringe, justamente, la ley. La seguridad de la tenencia puede ser obtenida a través de distintos instrumentos, desde tenencias intermedias hasta escrituras "duras" o completas.

A mediados de 2001 había en la región aproximadamente 128 millones de personas que vivían en tugurios, es decir, el 32\% de la población urbana. Se prevé que el número de habitantes en asentamientos precarios continuará en aumento si no se toman medidas preventivas y de regularización de la situación actual, complementadas con políticas que den acceso ordenado a la tierra urbana. Estos tugurios abarcan una amplia gama de asentamientos de bajos ingresos, desde viviendas deterioradas en el centro de la ciudad a los asentamientos informales con viviendas, infraestructura y servicios inadecuados y hacinados, ubicados en zonas de riesgo y con distintas formas de tenencia que en muchas ciudades, son la única opción posible para los pobres urbanos.

En general, el enfoque nacional sobre los tugurios ha variado, pasando de políticas negativas (desalojo, erradicaciones, negligencia benévola) a políticas más positivas como el mejoramiento de los asentamientos, la movilización de recursos locales para las mejoras y políticas basadas en el derecho. Se han aplicado distintas estrategias programáticas, desde las que se limitan a regularizar la propiedad de las viviendas irregularmente ocupadas, a programas integrados de mejoramiento de los barrios. Estas estrategias se fundamentan en un consenso regional generalmente aceptado de que las estrategias basadas en el asentamiento de poblaciones en las zonas que ya ocupan, proporcionan la solución más deseable desde el punto de vista social y económico al problema de los asentamientos informales. En este sentido, los asentamientos irregulares se aceptan como una realidad urbana que no puede erradicarse y se aceptan como parte del proceso de crecimiento de las ciudades.

La construcción social del hábitat por los habitantes de los asentamientos precarios y de los barrios consolidados debe ser considerada como un recurso y una inversión de los sectores de bajos ingresos que debe activarse, mejorarse o capitalizarse, es una de las principales oportunidades de desarrollo del hábitat en la región. En general los responsables por la formulación de políticas no reconocen en forma explícita este recurso ni su incorporación a las políticas de vivienda.

Con respecto a los programas de regularización de la tenencia, las complejas manifestaciones de la informalidad urbana del suelo, la multiplicidad de actores que intervienen, la falta de transparencia en lo que concierne a la propiedad del suelo y el enorme volumen de títulos que deben procesarse, constituyen serias dificultades para la implementación de estas iniciativas. Las estrategias de mejoramiento de los asentamientos informales incluyen inversiones para dotarlos de mejores infraestructuras y servicios urbanos, así como para desarrollar programas destinados a mitigar los principales problemas sociales de las comunidades y mejorar su calidad de vida en general. Se trata de programas de urbanización in situ que aprovechan las inversiones ya realizadas por los residentes en sus soluciones habitacionales y ponen énfasis 
en la participación comunitaria en la ejecución de las obras. Un aspecto económico y social crítico que no se ha incorporado plenamente en estos programas es la creación efectiva de oportunidades de generación de ingresos.

La escasez de tierras urbanizadas y la naturaleza especulativa de los mercados de tierras en las ciudades más urbanizadas de la región han provocado un notable aumento de los precios de la tierra en las ciudades de América Latina y el Caribe. Esta tendencia en las grandes ciudades ha tenido el efecto de relegar a los sectores de bajos ingresos a sus zonas periféricas debido al costo menor de la tierra y han contribuido a la segregación socio-espacial de los pobres. Se ha producido un incremento en la segregación en las ciudades de la región. Esta segregación toma distintas formas en las ciudades: desde barrios marginales en áreas consolidadas hasta extensas zonas tugurizadas en la periferia de las ciudades. Dentro de estas áreas también se encuentra una heterogeneidad en los niveles de ingresos. Por ejemplo, en Brasil, los no-pobres comprenden una proporción significativa de los residentes de favelas, hasta más de $50 \%$ en algunas ciudades.

La formación de asentamientos precarios en grandes ciudades ha reforzado los procesos de exclusión urbana y social, ha estimulado los conflictos en el uso del suelo en los planes de desarrollo urbano y ha obstaculizado los procesos de mejoramiento. La exclusión a menudo no se limita al acceso a los beneficios que la ciudad ofrece, sino también a la participación en las decisiones que afectan a la ciudad.

El proceso de urbanización ha permitido resolver algunas de las necesidades básicas de la población pero no ha podido facilitar la eliminación o substancial reducción de la pobreza. La informalización del mercado laboral debida a la incapacidad de las economías de generar suficiente empleo formal ${ }^{4}$, se considera entre las principales causas de la pobreza urbana. El sector informal ha llegado a representar el 73\% del mercado laboral urbano, especialmente en lo que se refiere a actividades de las prestaciones de servicios, que es el sector adonde se registra el mayor incremento. El desafío principal consiste en incorporar esta fuerza de trabajo en la economía formal, donde los niveles de salarios, protección social y productividad son más elevados. La insuficiencia de ingresos laborales como determinante básico de la pobreza urbana se ha abordado, en el corto plazo, en materia de diseńo de programas de capacitación laboral, provisión de crédito al sector informal, programas mixtos de empleo transitorio y capacitación focalizados en grupos vulnerables (mujeres, jóvenes, adultos mayores, grupos étnicos).

En la gran mayoría de los países de la región existen programas nacionales para facilitar el crédito, capacitar y entregar apoyo técnico a las pequeñas unidades productivas. Sin em-

\footnotetext{
4 La fuerte reducción de la capacidad de absorción de empleo de las industrias y del sector público en un contexto de acelerada incorporación de tecnología y de ajuste fiscal ha tenido al menos tres efectos: i) destrucción de puestos de trabajo de baja calificación, con aumento del desempleo y de la informalidad, ii) aumento de la brecha de salarios entre calificados y no calificados, y iii) disminución de la proporción de ocupaciones protegidas y estables, particularmente de los puestos de trabajo de baja calificación y aumento de los diferenciales entre calificados y no calificados en cuanto a los derechos asociados al trabajo (Kaztman, 2003).
} 
bargo, carecen de orientaciones claras 5 y tienen un marcado énfasis social, que no toma en consideración la enorme heterogeneidad que caracteriza el sector. La microempresa, bajo este esquema, se consolida como amortiguador laboral-productivo en tiempos de ajuste y crisis, sin necesariamente lograr insertarse y competir dentro de la economía formal.

Durante la última década ha habido un traslado de recursos y responsabilidades para el desarrollo de los programas de empleo a los municipios, o a otro tipo de jurisdicción menor. La experiencia municipal en esta materia demuestra logros limitados. Aunque los municipios intervienen en forma incipiente respecto a la demanda del empleo (el fomento productivo municipal), se encuentran fuertemente limitadas sus finanzas y capacidad de inversión. El desarrollo de un apoyo hacia la oferta (a los recursos y capacidades de trabajo con que cuentan personas y hogares pobres) ha sido más esporádico, y sin articulación con los esfuerzos sobre la demanda. Los programas públicos de intervención en la precariedad del hábitat en general no han incorporado la temática del empleo, aunque sí se detectan algunos casos, por ejemplo en Chile y Argentina.

\section{La pobreza urbana y los mercados laborales}

A partir de 1990, el empleo en las áreas urbanas mostró una mayor expansión en los sectores de baja productividad. En la región en su conjunto, aumentó la participación del sector informal en el empleo urbano, de un $42,8 \%$ en 1990 a un $46,7 \%$ en el 2003. De hecho, se incrementó la participación en la composición del empleo urbano de todas las categorías de empleo informal, es decir del trabajo por cuenta propia no profesional, administrativo o técnico, incluido el trabajo familiar no remunerado, el empleo en microempresas y el servicio doméstico. En cambio, se redujo la participación del empleo tanto en las pequeñas, medianas y grandes empresas privadas como en el sector público (CEPAL, 2005).

Durante la década de los noventa continuaron manifestándose los procesos de urbanización de la fuerza de trabajo, de aumento de la cantidad de personas en edad de trabajar y de alza de las tasas de actividad económica de la mujer. Estudios demuestran (Banco Mundial, 2004) que las mujeres tienden a integrarse a los mercados laborales en trabajos de mayores niveles de inseguridad laboral, y de tiempos parciales, aparentemente permitiéndoles combinar el cuidado infantil con un trabajo estructurado.

Ha continuado el desplazamiento de empleos desde el sector de producción de bienes hacia el área de servicios. También, se acentúa la precariedad laboral: las contrataciones de corta duración (temporal, estacional o a tiempo parcial), la ampliación de las causales de término de contrato, la reducción de las indemnizaciones por despido, la limitación del derecho a huelga y poco acceso a los servicios de la seguridad social.

\footnotetext{
5 Los países de la región carecen de políticas nacionales de fomento a la pequeña producción que incorpore una perspectiva de desarrollo económico local.
} 
Se ha visto un aumento en la tasa de desempleo en áreas urbanas. Es una tendencia reciente en América Latina y es un problema crítico para el grupo de países de Argentina, Colombia, Panamá, República Dominicana, Uruguay y Venezuela donde, en 2002, las tasas son mayores al 15\%. Un segundo grupo de países lo constituyen Brasil y Chile, donde el desempleo, a principios de la década de 2000, alcanza a más de un 10\% (CEPAL, 2005).

\section{Pobreza urbana e informalidad laboral}

Gran parte de los jefes de hogares urbanos, sean indigentes, pobres o no pobres, laboran en el sector informal ${ }^{6}$. Si bien la informalidad laboral es más extendida entre los hogares pobres e indigentes, ella también está presente en el $43 \%$ de los hogares no pobres. La comparación de cifras de comienzos y finales de la década pasada señalan que el empleo informal ha seguido aumentando. Los indigentes, que ya en 1990 dependían en un 68\% del empleo informal hoy lo hacen en un $70 \%$. Por otra parte, aunque en los hogares no pobres los jefes de hogar aún trabajan mayoritariamente en el sector formal, la informalidad también aumenta. En total, de acuerdo a estudios recientes, se han sumado más de 3.000.000 de hogares que dependen del sector informal, a los 9.600.000 que ya existían en 1990 (Mac Donald, 2004).

La informalidad se mantiene alta a inicios de esta década y cada vez hay mayor concentración del empleo en el sector de los servicios, en una tendencia persistente desde los años noventa en América Latina y el Caribe. La importancia del sector informal como fuente de trabajo e ingresos, particularmente para los pobres, es evidente. Sus ingresos por ocupado en general son bajos e inferiores a los que se obtienen en el sector formal (aunque en el sector formal los pobres generalmente también tienen empleos inseguros de rentabilidad baja (Banco Mundial, 2004)). Ello refleja, en parte, diferencias de capital humano pero, principalmente, de las características productivas de las unidades que componen el sector (escasos requerimientos de capital y tecnología, y por ende, resultan en baja productividad e ingresos por persona ocupada) y de los mercados a los que tienen acceso.

El sector informal en la actualidad contiene en su interior un conjunto heterogéneo de actividades con distintos grados de funcionalidad y con resultados diversos sobre los ingresos y la productividad. Esta diversidad de situaciones es importante de considerar para el diseño de políticas; pero para el conjunto del sector prevalece el desequilibrio estructural entre excedente de mano de obra y disponibilidad de empleos, como factor determinante de homogeneización relativa a bajos niveles de ingreso (Tokman, 2004).

Aunque por una parte la economía latinoamericana no proporciona suficientes empleos, por otra parte, algunas características de los pobres urbanos hacen más difícil que accedan a los pocos buenos trabajos existentes. En particular, un $70 \%$ de los adultos pobres tienen

\footnotetext{
6 Se utiliza la clasificación elaborada por la División de Estadísticas de CEPAL, de "empleo formal” y “empleo informal”, aplicada en este caso a los jefes de hogar. Más allá de las particularidades en cada país y entre rondas de encuestas, se considera como empleo informal al empleo no calificado en microempresas (aquellas cuyo variable tamańo del establecimiento tiene un número igual o menor a 5 trabajadores), al empleo doméstico y al trabajo independiente no calificado de cualquier rama de productiva.
} 
bajos niveles de habilidades, contra un 50\% para la fuerza de trabajo urbana en su totalidad. Esta situación se debe a niveles menores de educación, pero también a la calidad deficiente de educación y de capacitación a la cual los pobres urbanos generalmente pueden acceder. Existen variaciones entre países: Argentina, Chile y Colombia con más de un quinto de los jefes de hogares pobres con a lo menos 11 años de escolaridad (Urani, 2003). Asimismo, en el caso de niveles de remuneración, la evidencia demuestra que diferencias en salarios entre distintos grupos de personas pueden adscribirse a diferencias en educación (Arias, Yamada y Tejerina, 2003).

\section{Precariedad del hábitat e informalidad laboral ${ }^{7}$}

En la mayor parte de los países de la región la pobreza urbana habita de manera formal y al mismo tiempo subsiste gracias al sector informal. Esta constatación debería tenerse muy en cuenta al revisar los programas de superación de la precariedad urbana que se aplican en la región, ya que éstos en su mayoría se concentran en los llamados campamentos, tugurios, etc., sin considerar que la pobreza se encuentra en su mayor proporción "larvada" en barrios consolidados originados en programas públicos de vivienda o en antiguas invasiones hoy consolidadas. Otro grupo importante es el que presenta una pobreza "formal" en ambas dimensiones de empleo y hábitat. La experiencia de algunos países parece indicar que la pobreza formal es especialmente vulnerable a los ciclos económicos, tanto por los costos de vivir en viviendas convencionales como por la mayor rigidez que enfrentan los hogares a la hora de ajustar sus patrones de vida cuando lo requieren las fluctuaciones en el ingreso.

Es significativo que en esta visión agregada de la informalidad residencial, ningún país presente una proporción importante de pobres urbanos que residen de manera informal y cuentan con empleos formales. Este "casillero vacío" parece indicar que de alguna forma, los pobres urbanos están formalizando su hábitat, pero siguen teniendo serias barreras para lograr una inserción laboral adecuada en esta región. Cabe comentar en este caso la acelerada adopción por parte de las políticas de vivienda de los países latinoamericanos, cuyos esquemas de acceso a la vivienda y a otros bienes urbanos presuponen que los postulantes tengan ingresos regulares para hacer frente a compromisos de ahorro y crédito. Considerando que los pobres urbanos informales, que deberían ser los sujetos preferentes de estas políticas, subsisten en la mayoría de los países gracias a la economía informal.

Es importante agregar que por la distribución espacial de los pobres urbanos a la periferia de los conos urbanos (en muchas ciudades de la región), y una distribución espacial del empleo a otros sectores de la ciudad (centros históricos por el comercio ambulante; barrios residenciales de sectores de ingresos altos; barrios industriales), se ha aumentado los costos de transporte para los pobres urbanos, como factor adicional en la búsqueda de empleo.

Asimismo, los pobres urbanos, por vivir en barrios "pobres", son frecuentemente discriminados en el mercado laboral, por provenir de barrios estigmatizados. 


\section{Estrategias públicas para atender la vulnerabilidad económica de los pobres urbanos}

Revisaremos las estrategias públicas actuales para atender a la vulnerabilidad económica de los pobres urbanos, desde el campo del hábitat y la intervención urbano-territorial. Antes de profundizar en la experiencia en la región en esta temática, es importante recalcar que políticas orientadas a fomentar un crecimiento económico elevado y sostenido, así como un desarrollo productivo fundado en el aumento de la productividad y la incorporación continua de innovaciones, son claves para la generación del empleo productivo ${ }^{8}$.

Las políticas del hábitat están incorporando, de manera aún incipiente, una preocupación por facilitar el acceso de los pobres urbanos al empleo y a ingresos estables. Inicialmente, esta inquietud ha sido más bien general, y se ha expresado en reflexiones e iniciativas tendientes a lograr que las ciudades sean más competitivas y que aumente la productividad urbana.

\section{La productividad urbana}

Bajo esta comprensión de la problemática, la ciudad es entendida en su potencialidad productiva, y no solamente vista en términos de consumo. Se postula que ciudades dinámicas y "sanas" son una parte integral de un crecimiento económico sostenido. Al desarrollarse un país, las ciudades contribuyen en una mayor proporción, y en forma creciente, al ingreso nacional. De acuerdo al Banco Mundial (Vidler, 1999), las áreas urbanas de los países de bajos ingresos generan un $55 \%$ del producto interno bruto; de los países de ingresos medios, un $73 \%$ y las áreas urbanas de los países de ingresos altos, un $85 \%$.

Teóricamente, estas estrategias se fundamentan en que las ciudades proporcionan economías de aglomeración tanto por localización (los beneficios generados por la proximidad a empresas del mismo sector o rubro) como por urbanización (los beneficios generados por la proximidad a muchos actores económicos distintos) y que éstas aumentan la productividad de las actividades económicas en áreas urbanas. Asimismo, estudios del Banco Mundial muestran que la productividad de empresas localizadas en áreas urbanas aumenta con el tamaño de ciudad (Vidler, 1999). Para explotar los beneficios de aglomeración, las ciudades pueden (Vidler, 1999) aumentar la inversión en capitales (infraestructura, vivienda, social) a través de un financiamiento tanto público (multi-nivel) como privado. Asimismo, utilizar instrumentos de uso de suelo (zonificación) para racionalizar los usos competitivos de éste, minimizar externalidades negativas y dinamizar procesos de desarrollo. Finalmente, los gobiernos pueden influir sobre el tipo de desarrollo con la ubicación de obras públicas (tanto pequeñas como grandes). También tienen un rol clave como depositario de derechos de propiedad a través de los registros prediales, de los procesos de catastro y de la información sobre el mercado de suelo. Por último, los gobiernos locales pueden entablar estrategias de entrepreneur público, buscando

\footnotetext{
8 Para profundizar estos tema ver (1) CEPAL (2005). Estudio económico de América Latina y el Caribe, 2004 - 2005, (2) CEPAL (2004b), Desarrollo productivo en economias abiertas, y (3) Stallings, B. y J. Weller (2003), La generación de empleo en América Latina en los años noventa: base fundamental de la política social, en A. Puyana y G. Farfán, coord., Desarrollo, Equidad y Ciudadanía. Las políticas sociales en América Latina, México, D.F: FLACSO, Plaza y Valdés, 143-180.
} 
proveer la ciudad de un ambiente adecuado (minimizar costos de transporte, calidad de vida, etc.), minimizar la burocracia local hacia las empresas y fomentar políticas sectoriales hacia el empresariado, por ejemplo, foros de concertación facilitando información de calidad.

Complementario a esta perspectiva, es la propuesta de Amis (1999) sobre el papel de los gobiernos locales respecto a la pobreza y el crecimiento económico en las ciudades. Los gobiernos locales deben cumplir funciones tales como: facilitar la participación de los pobres en el crecimiento económico y amortiguarles a ellos los efectos de recesiones o ajustes. De acuerdo a estos estudios, un factor clave dentro del ámbito del gobierno local es que no tenga estructuras y sistemas de mala governance (servicios colectivos insuficientes, sistema fiscal inadecuado o implementado parcialmente, etc.).

Estudios respecto a las estrategias de desarrollo urbano que logran combinar metas de aumento en productividad con mejoras en condiciones de equidad demuestran la importancia de maximizar las fortalezas específicas en cada ciudad. En dos casos brasileños (Toledo y Cambe), los alcaldes lograron impulsar un crecimiento económico local a través de la identificación y capitalización de ventajas existentes, para movilizar y maximizar todos los recursos locales posibles (Fergusen, 1992). En Toledo, el alcalde (previamente Presidente de la Asociación de Comercio e Industria de Toledo) capitalizó el "espíritu colectivo" desarrollado en una iniciativa de industrias comunitarias fomentado por la Alcaldía. Aunque la iniciativa falló, la confianza y voluntad comunitaria generada por la experiencia alimentó un proceso de modernización del sistema de patentes de la administración local en conjunto con un proceso de formalización de empresas locales. Con la generación de estos recursos adicionales, se logró, trabajando en conjunto con las organizaciones comunitarias, una expansión significativa de servicios a barrios pobres. En Cambe, fue un liderazgo y apoyo fuerte a empresas emergentes, que proporcionó empleo y recursos para aumentar servicios para los sectores pobres urbanos.

\section{Experiencias desde el campo del hábitat}

Existen pocas experiencias en la región que logran integrar las metas de mejorar la habitabilidad y las oportunidades productivas de los habitantes de barrios precarios. En general, los acercamientos son parciales, sin la instalación de modelos de desarrollo sostenible local. Al nivel regional, se detecta algunas experiencias de potenciar las posibilidades existentes en las ciudades y los barrios precarios, para que los hogares de escasos recursos tengan acceso a espacios e instalaciones adecuadas para el fomento productivo local y la generación de oportunidades de ingreso. El Cuadro 2 presenta una serie de iniciativas en la región que ejemplifican estrategias multipropósitos en el campo del empleo, el hábitat y los ingresos. 
Cuadro 2. Estrategias multipropósitos en América Latina y el Caribe (empleo, hábitat e ingresos)

\begin{tabular}{|c|c|}
\hline Tipología de intervención & Experiencias \\
\hline \multirow[t]{3}{*}{$\begin{array}{l}\text { Vivienda, asentamientos precarios y espacios } \\
\text { productivos }\end{array}$} & $\begin{array}{l}\text { - Programa Vila de Oficios, Curitiba, Brasil. En favelas, provisión de viviendas } \\
\text { de dos plantas, una dedicada a generación de ingresos. Proyecto ejecutado por } \\
\text { municipio y ONG locales. Integrado a programa de urbanización. }\end{array}$ \\
\hline & $\begin{array}{l}\text { - Programa de Financiamiento de Vivienda (Bolivia). Vivienda Social - Productiva: } \\
\text { Subsidio parcial para la mejora o ampliación de viviendas en ambientes destina- } \\
\text { dos a usos productivos. Coparticipan en este subprograma los municipios y el } \\
\text { beneficiario. }\end{array}$ \\
\hline & $\begin{array}{l}\text { - Programa de Mejoramiento de la Comunidad (Paraguay). Incorpora la creación } \\
\text { de espacios de producción a nivel comunitario. }\end{array}$ \\
\hline \multirow[t]{2}{*}{$\begin{array}{l}\text { Impulsar la creación de zonas empresariales } \\
\text { cercanas a sectores de pobreza, y articuladas } \\
\text { con programas habitacionales }\end{array}$} & $\begin{array}{l}\text { - Programa Mini distritos industriales y de servicios, Sao José do Río Preto, Sao } \\
\text { Paolo, Brasil. En conjunto con loteos habitacionales, designación e incentivos para } \\
\text { instalación de empresas, en sectores de favelas. Ejec. por municipalidad (a). }\end{array}$ \\
\hline & $\begin{array}{l}\text { - Villa El Salvador, Lima, Perú (b). Parque industrial orientado a pobladores de } \\
\text { asentamientos precarios cercanos (a). }\end{array}$ \\
\hline $\begin{array}{l}\text { Promoción del uso de métodos de trabajo- } \\
\text { intensivo en la construcción de vivienda, } \\
\text { infraestructura y equipamiento (más capa- } \\
\text { citación en oficios) }\end{array}$ & $\begin{array}{l}\text { - Programa Federal de Emergencia Habitacional (Argentina). Formación y capaci- } \\
\text { tación de Cooperativas de Vivienda de personas cesantes para la autoconstrucción } \\
\text { asistida de viviendas; contratación por Municipios y otros. }\end{array}$ \\
\hline $\begin{array}{l}\text { Incorporación en los programas de mejora- } \\
\text { miento de asentamientos precarios, apoyo a } \\
\text { microemprendimientos y capacitación laboral } \\
\text { para sectores pobres urbanos }\end{array}$ & $\begin{array}{l}\text { - Programa Rosario Hábitat (Argentina). Integrar capacitación en oficios para jóvenes } \\
\text { e incubadores para microemprendimientos en programa integral de mejoramiento } \\
\text { de asentamientos irregulares en Ciudad de Rosario. }\end{array}$ \\
\hline $\begin{array}{l}\text { Incorporación del ahorro popular colectivo en } \\
\text { los programas de mejoramiento de barrios, y } \\
\text { de generación de micro-emprendimientos }\end{array}$ & $\begin{array}{l}\text { - Banco popular PALMAS, Palmeira, Fortaleza, Brasil. Banco popular en favela } \\
\text { con esquemas de ahorro y crédito (productivos; emergencia; mejoramiento hábitat; } \\
\text { vivienda). Implementa municipio, asociaciones vecinales, ONG, GTZ (a). }\end{array}$ \\
\hline $\begin{array}{l}\text { Fortalecer programas de administración, } \\
\text { financiera, comercial y productiva para sectores } \\
\text { de subsistencia en barrios precarios }\end{array}$ & $\begin{array}{l}\text { - Programa de generación de empleo y renta, Quizadá, Ceará, Brasil. Crédito popular } \\
\text { productivo; Asistencia Técnica a entidades productivas; formación de cooperativas } \\
\text { y asociaciones; capacitación de mano de obra; regularización del sector informal. } \\
\text { Ejecutado por municipio y articulado con otros programas sociales (a) }\end{array}$ \\
\hline
\end{tabular}

Fuente: Elaboración propia.

Nota: (a) Para mayores informaciones, ver POLIS (2002). (b) En Villa El Salvador, Lima, Perú, la intención de proveer espacios para el trabajo se incorporó desde el origen de este asentamiento de 324.000 habitantes, que logra en 1971 que se legalice la invasión de terrenos que le dio origen. Para ello, la planificación inicial de la invasión contempló terrenos para establecer un parque industrial. Sin embargo, el desarrollo posterior de Villa El Salvador no logró atraer una actividad industrial significativa. Para activar el proyecto, se crea a mediados de los años 90 una Oficina de Desarrollo Local y un Centro de Desarrollo Empresarial. Los industriales se encuentran asociados, y hoy la Autoridad Autónoma del Parque Industrial es una figura público-privada. Pese a la discontinuidad del proyecto asociada a fluctuaciones de apoyo político y a una escasa capacidad técnica de la municipalidad para apoyar el proceso, se han generado más de 12.000 empleos en esta zona en que habitan familias de escasos recursos.

Programas que combinen acciones multipropósito exigen arreglos institucionales complejos que exceden el ámbito sectorial. En los países de la región, se han aplicado diversos modelos organizativos para ejecutar estos programas, entre los que destacan aquellos que incorporan a la institucionalidad local y a la comunidad de manera decidida. Sobre la base de un registro de las diversas formas institucionales existentes y de la manera en que se enlazan entre ellos 
el nivel nacional con el local, sería posible identificar las modalidades más convenientes para lograr una efectiva masificación de los programas de superación de la pobreza y precariedad en las ciudades. Los nuevos modelos de gestión muestran también una apertura mayor a la participación del sector privado, ONG, bancos, cooperativas, etc. Este tipo de participación todavía necesita de una adecuada regularización y control por parte del sector público, que sigue teniendo un rol fundamental y clave en la gestión de las políticas habitacionales y urbanas (Simioni y Szalachman, 2006).

Otro aspecto fundamental que se destaca es que la formulación de programas que ofrezcan una diferenciación de la oferta habitacional para distintos sectores en la misma área, intervenciones en la provisión de infraestructura y en la conexión con las redes viales, y creación de espacios públicos, juegan un papel fundamental en materia de prevención y/o mitigación de las situaciones de aislamiento y exclusión que afectan a las personas de menores recursos, y les permiten, especialmente a los jóvenes, salir de su propio barrio y entrar en contacto con otras realidades urbanas. Esto pudiese favorecer el desarrollo de capital social y humano.

A fin de lograr el crecimiento económico urbano a favor de los pobres, es necesario reconocer la contribución del sector informal al desarrollo económico y al empleo. Pese a que las empresas informales tienen un estatus no reconocido y no están sujetas a regulaciones adecuadas en materia de producción y empleo, producen y distribuyen bienes en los mercados y tienen numerosas interdependencias con la economía formal. La apreciación de estas empresas desde la perspectiva de la política pública muestra cierta ambigüedad dado que, por una parte, constituyen formas apreciadas de sustento para grupos vulnerables y son un semillero de capacidad empresarial pero, por otra, configuran ámbitos de la economía en los que, muchas veces, la falta de normatividad y regulación atenta contra el bien común y la rentabilidad de empresas formales?

Ante el aumento del desempleo y el empleo informal, algunos países y ciudades han tomado conciencia de que el empleo informal de subsistencia puede ser por el momento la única alternativa viable que encuentran los hogares pobres para sobrevivir o salir de la pobreza, por lo que las iniciativas urbanas deberían cuidar que él sea posible mientras no surjan otras posibilidades de mayor productividad. Una preocupación especial, en este sentido, son los comerciantes ambulantes. Varias ciudades en la región durante los ańos noventa implementaron diversas estrategias para la re-localización del comercio ambulante en el marco de los planes de desarrollo urbano (Quito, Lima, Kingston, Santiago, Bogotá y Río de Janeiro). Estas experiencias han sido relativamente exitosas en la medida que han logrado los resultados esperados: reordenamiento urbano, uso adecuado del espacio público y mejora o mantenimiento del empleo en los vendedores ambulantes a pesar de la reubicación. En Quito, el trabajo con los comerciantes se ubicó dentro de un programa integral de restauración del casco histórico central de la ciudad (Cuadro 3). En Bogotá, se coordina la creación de espacios públicos productivos para el comercio callejero con el diseño de la infraestructura de transporte.

9 Para mayores informaciones sobre este tema ver, CEPAL (2004b), Desarrollo productivo en economías abiertas, pp. 247-249. 
Cuadro 3. Resumen. Plan de reubicación y modernización del comercio minorista del centro histórico de Quito (Ecuador)

\begin{tabular}{|c|c|}
\hline \multirow{4}{*}{$\begin{array}{c}\text { Antecedentes } \\
\text { generales }\end{array}$} & · Población Quito: 1.500 .000 habitantes. \\
\hline & $\begin{array}{l}\text { Centro histórico de Quito: patrimonio cultural de la humanidad UNESCO 1978, símbolo patrimonio } \\
\text { histórico-identitario significativo, población } 80.000 \text { habitantes, principalmente de escasos recursos, y gen- } \\
\text { trificación; contracción residencial entre } 1990 \text { y } 2000 \text { y aumento notorio de su función comercial; arraigo } \\
\text { cultural en el comercio callejero de mercado. }\end{array}$ \\
\hline & . 32\% PEA en Quito en sector informal (1997). \\
\hline & $\begin{array}{l}\text { Proyecto BID-Municipio Quito: se estableció la Empresa de Desarrollo del Centro Histórico de Quito. } \\
\text { Con el municipio, se desarrolla Administración de la Zona Centro, y el Plan Maestro para el Comercio } \\
\text { Minorista con implementación de permisos municipales }(1994-1998) \text {. }\end{array}$ \\
\hline \multirow[t]{4}{*}{$\begin{array}{l}\text { Antecedentes } \\
\text { de problema }\end{array}$} & $\begin{array}{l}\text { Concentración alta de comercio minorista (callejero) y ambulante ( } 8000 \text { en } 22 \text { manzanas + } 2000 \text { en zona } \\
\text { central; promedio } 140 \text { /cuadra). }\end{array}$ \\
\hline & $\begin{array}{l}\text { - Congestión peatonal y callejera; población afectada por situación: } 320.000 \text { visitantes, residentes, } 40.000 \\
\text { propietarios de locales comerciales, } 183 \text { escuelas, } 5000 \text { edificaciones patrimoniales, } 362 \text { monumentos his- } \\
\text { tóricos, comerciantes informales, turismo. }\end{array}$ \\
\hline & . $70 \%$ comerciantes agrupados en 96 asociaciones. \\
\hline & $\begin{array}{l}\text { Deterioro de la imagen urbana e infraestructuras de la ciudad: contaminación visual, contaminación } \\
\text { ambiental, deterioro de espacios y bienes públicos urbanos y apropiación privada, riesgo urbano ambiental } \\
\text { (bodegas, contaminación, condiciones sanitarias inadecuadas), congestión vehicular (velocidad menor a } \\
\text { peatonal), violencia y crimen organizado. }\end{array}$ \\
\hline \multirow[t]{5}{*}{ Objetivos } & . Garantizar mejores condiciones de vida y dignidad para los comerciantes y consumidores. \\
\hline & . Recuperar el espacio público. \\
\hline & . Preservar el Centro Histórico. \\
\hline & . Reubicar y descentralizar el comercio callejero a Centros Comerciales Populares. \\
\hline & $\begin{array}{l}\text { Crear el ambiente y seguridad adecuados para impulsar y desarrollar el turismo como la mayor fuente de } \\
\text { productividad del Distrito Metropolitano. }\end{array}$ \\
\hline \multirow[t]{2}{*}{ Componentes } & $\begin{array}{l}\text { Estrategias de concertación, negociación, participación, comunicación y socialización, en } 3 \text { programas } \\
\text { orientados al comercio popular de: desarrollo urbano territorial, desarrollo político institucional y desarrollo } \\
\text { socio-económico. }\end{array}$ \\
\hline & $\begin{array}{l}\text { Contempla actividades de: construcción y readecuación de nuevos Centros Comerciales Populares en } \\
\text { distintas zonas de Quito, apoyo a la administración de los Centros, capacitación, plan de ME para la limpieza, } \\
\text { mecanismos de adjudicación de locales y legalización de títulos, estrategias de publicidad y comunicación. }\end{array}$ \\
\hline Resultado & $\begin{array}{l}\text { Reconciliación del derecho al trabajo de la población de escasos recursos (y mejoramiento de su calidad } \\
\text { de vida) con el derecho a la disponibilidad y acceso a los espacios públicos, y a su vez, el uso estratégico del } \\
\text { recurso patrimonial para el desarrollo urbano. }\end{array}$ \\
\hline
\end{tabular}

Fuente: Proyecto "Pobreza urbana: estrategia orientada a la acción para los gobiernos e instituciones municipales en América Latina y el Caribe", realizado por CEPAL entre 2003 y 2005.

Respecto a la perspectiva del desarrollo económico local, en general, los países de la región carecen de políticas nacionales para la promoción de la producción en pequeña escala. Las experiencias municipales en este sentido han tenido poco éxito, aunque reflejan una creciente demanda (Llorens, Alburquerque y Castillo, 2002). Una experiencia notable es la de PRODEL en Nicaragua.

\section{Conclusiones y opciones de política}

La calidad de los ingresos reales de los sectores de pobreza urbana tiene directa e indirecta relación con la posibilidad de mejorar su situación de vida. Para muchas de las condiciones que caracterizan la pobreza urbana en nuestra región, la magnitud y estabilidad de los ingresos influyen tanto en forma directa como indirecta sobre las posibilidades de superar o mitigar la 
condición. Por ejemplo, la calidad de la vivienda y la seguridad de la tenencia se relacionan con la capacidad de pago (tanto dentro de los mercados formales como los informales); aunque la estrategia de autoconstrucción permite soluciones precarias fuera del mercado, la inseguridad de la tenencia prevalece, muchas veces en conjunto con la inseguridad urbano-ambiental, hechos que refuerzan negativamente la inestabilidad de la cartera de activos materiales de los hogares urbanos pobres. Asimismo, el acceso seguro a la infraestructura colectiva (de agua potable, saneamiento, drenaje, calles, espacio público) también tiene relación con la capacidad de pago, y la falta de educación influye sobre ingresos a futuro.

Durante las últimas décadas, las políticas del hábitat han ido incorporando una marcada orientación hacia la economía de mercado en sus programas. Desde el punto de vista de la oferta se exigen a los sectores de pobreza ahorros, pagos para servicios, entre otros. Esta estrategia de política sin lugar a duda, ha ayudado a ampliar la cobertura de estos programas, diversificando las formas de financiar la inversión pública, como también ha ayudado a facilitar la asimilación de una cultura relacionada a esta economía. Sin embargo, la comprensión de esta realidad de economía de mercado, desde el punto de vista del hogar (e individuo), ha sido ajena a la política habitacional y a la intervención urbano-territorial. Esta falla en el marco de las políticas del hábitat en la región no ha permitido potenciar a los sectores de pobreza en aspectos críticos de su desarrollo. Asimismo, ha inhibido a que la política pública comprenda su potencialidad "destructiva" sobre los activos y posibilidades de desarrollo de los pobres urbanos (por ejemplo, construcción de vivienda social en lugares apartados de fuentes de trabajo y sin redes de transporte adecuadas y accesibles).

A nivel de política de hábitat, y en especial en lo que se refiere a la vivienda, la tenencia, los servicios colectivos, la segregación urbano-territorial, y el mejoramiento integral de barrios precarios, se requiere transversalizar las implicaciones de la vulnerabilidad económica de los sectores de pobreza urbana en el diseño de los programas. En tanto, estos programas no deben erosionar la cartera de activos que ya han adquirido estos sectores. Al contrario, los programas deben buscar maneras para potenciar y ampliar los activos de los pobres urbanos, como también buscar sinergias entre fortalecer distintos tipos de activos.

Desde la perspectiva de potenciar a los sectores de pobreza urbana como protagonistas de su propio desarrollo, se recomienda entablar una agenda del hábitat para atender la vulnerabilidad económica de los pobres urbanos. En este contexto, se debiera buscar el mejoramiento de las condiciones del hábitat, entendiendo que éstas forman parte de la dotación de capital físico y económico de los sectores urbanos de pobreza. Como segundo elemento, se debiera mejorar en la dotación de capital humano y social de los grupos pobres urbanos en relación a su dimensión económica. Y finalmente, se debiera preservar y mejorar la dotación de los capitales naturales a través de la conservación del medio ambiente urbano y reducción de la vulnerabilidad urbana. Esta agenda es consistente con los principios del desarrollo sostenible, y en este sentido, abarca las dimensiones ambientales, económicas y sociales del desarrollo.

En resumen, una agenda del hábitat para atender la vulnerabilidad económica de los pobres urbanos debiera buscar por un lado mejorar (calidad, reducción de barreras de acceso) la cartera 
de activos (capital humano, capital social, capital productivo) 10 de los pobres urbanos en su dimensión económica (Cuadro 4), y por otro, mejorar niveles o estabilidad de ingresos de los pobres urbanos (en forma directa e indirecta).

Cuadro 4. Estrategias y acciones para fortalecer la cartera de activas de los sectores de pobreza urbana.

\begin{tabular}{|c|c|}
\hline \multirow{5}{*}{$\begin{array}{l}\text { Capital } \\
\text { humano }\end{array}$} & Incorporar la capacitación vocacional en los programas de mejoramiento integral de barrios. \\
\hline & $\begin{array}{l}\text { Incorporar la capacitación en oficios de construcción (social) y producción de materiales de construcción en } \\
\text { políticas de hábitat. }\end{array}$ \\
\hline & $\begin{array}{l}\text { Articular con programas de habilitación social en los programas de mejoramiento integral de barrios (habilidades } \\
\text { y hábitos orientados al trabajo). }\end{array}$ \\
\hline & $\begin{array}{l}\text { Incorporar el cuidado infantil en barrios locales en programas de hábitat y/o articulación local con otros } \\
\text { programas sociales. }\end{array}$ \\
\hline & $\begin{array}{l}\text { Mejorar el acceso a redes e infraestructura de transporte urbano (acceso físico vía desarrollo de infraestructura y } \\
\text { redes de transporte, y mayor integración de nueva vivienda social en la trama urbana, y acceso por precio). }\end{array}$ \\
\hline \multirow{4}{*}{$\begin{array}{l}\text { Capital } \\
\text { social }\end{array}$} & Incorporar el ahorro popular colectivo en los programas de mejoramiento de barrios, y de vivienda social. \\
\hline & $\begin{array}{l}\text { Incorporar programas orientados al sector informal y el uso del espacio público en los programas de hábitat, } \\
\text { especialmente a través de las asociaciones de comerciantes (informales y formales). Articulación con programas } \\
\text { municipales de este mismo. }\end{array}$ \\
\hline & $\begin{array}{l}\text { Articulación/incorporación de programas de micro-emprendimientos en esquemas grupales en los programas } \\
\text { de hábitat. }\end{array}$ \\
\hline & Fortalecer organizaciones sociales y sus vínculos con institucionalidad formal local. \\
\hline \multirow{4}{*}{$\begin{array}{c}\text { Capital } \\
\text { productivo }\end{array}$} & $\begin{array}{l}\text { Asegurar la tenencia de la vivienda social y viabilidad de derechos de propiedad para que la vivienda sirva como } \\
\text { activo para el ahorro intergeneracional, y "savings safety net" en tiempos de emergencia. }\end{array}$ \\
\hline & $\begin{array}{l}\text { Promover la utilización de la micro-finanzas en (i) el mejoramiento de viviendas para que incorpore actividades } \\
\text { productivas y/o oportunidades de arriendo, y (ii) proyectos de generación de ingresos dentro de los proyectos de } \\
\text { mejoramientos de barrios, incorporando la producción de materiales de construcción, y desde una perspectiva } \\
\text { de la construcción social del hábitat. }\end{array}$ \\
\hline & Promover la construcción de vivienda social con espacios productivos. \\
\hline & mercio de micro-escala. \\
\hline
\end{tabular}

Fuente: Elaboración propia.

Respecto al segundo objetivo de desarrollo, se debiera crear oportunidades empresariales (micro, pequeña y mediana), y de empleo para los sectores de pobreza en áreas urbanas:

- Promover el uso de métodos de trabajo-intensivo en la construcción de vivienda, infraestructura y equipamiento.

- Favorecer en las licitaciones públicas los servicios de los pequeños y medianos emprendimientos de propietarios de sectores de vulnerables.

- Fortalecer programas de administración, financiera, comercial y productiva para la micro, pequeña y mediana empresa.

- Impulsar la creación de zonas empresariales cercanas a sectores de pobreza.

\footnotetext{
10 Activos que una persona posee como consecuencia de las características propias de su condición humana: conocimiento, salud, destrezas, tiempo, y otros; activos que se tienen como consecuencia de las relaciones de una persona con otros y la participación en organizaciones (tales relaciones facilitan el acceso a otros recursos); activos físicos y activos financieros, respectivamente.
} 
- Diseñar y promover políticas de inversión que permitan la existencia de infraestructura comercial a la cual tengan acceso los comerciantes ambulantes.

- Impulsar programas integrales de mejoramiento de hábitat y de oportunidades productivas para asentamientos precarios.

- Impulsar el desarrollo de estrategias de desarrollo urbano que permitan integrar las metas de funcionalidad urbana con la habitabilidad de los pobres urbanos, en un contexto de fomento al empleo con base territorial.

\section{Referencias bibliográficas}

Amis, P. (1999). Urban economic growth and poverty reduction. Theme paper No2, ESCOR, Urban governance, partnership and poverty. Birmingham: International Development Department, School of Public Policy, University of Birmingham.

Arriagada, I. (2005). Dimensiones de la pobreza y políticas desde una perspectiva de género. Revista de la CEPAL, 85, 101-113.

Arriagada, I.; Miranda, F. \& Pavez, T. (2004). Lineamientos de acción para el diseño de programas de superación de la pobreza desde el enfoque del capital social. Guía conceptual y metodológica. Serie Manuales, 36. Santiago de Chile: División de Desarrollo Social, Comisión Económica para América Latina y el Caribe, Naciones Unidas.

Banco Mundial (2004). The urban poor in Latin America. Report No 30465. Finance, Private Sector and Infrastructure Management Unit, Latin America and the Caribbean Region.

CEPAL (2004a). Panorama social de América Latina 2004. Santiago de Chile: Naciones Unidas.

CEPAL (2004b). Desarrollo productivo en economias abiertas. Santiago de Chile: Secretaría ejecutiva, Naciones Unidas.

CEPAL (2005). Estudio económico de América Latina y el Caribe, 2004 - 2005. Santiago de Chile: Naciones Unidas.

CEPAL (2007). Panorama social de América Latina 2007. Santiago de Chile: Naciones Unidas.

Comisión Económica para América Latina y el Caribe (CEPAL) (2000). Equidad, desarrollo y ciudadanía. Bogotá: Editorial Alfaomega.

Fergusen, B.W. (1992). Inducing local growth: two intermediate-sized cities in the State of Parana, Brazil. Third World Planning Review, 14, 3, 245-265.

Foro Internacional de Pobreza Urbana (FIPU) (1996). Urban poverty: a world challenge. The Recife declaration. París: United Nations Centre for Human Settlements.

Kaztman, R. (2003). La dimensión espacial en las políticas de superación de pobreza. Serie Medio Ambiente y Desarrollo, 59. Santiago de Chile: División de Desarrollo Sostenible y Asentamientos Humanos, CEPAL, Naciones Unidas.

Llorens, J., Alburquerque, F. \& Del Castillo, J. (2002). Estudio de casos de desarrollo económico local en América Latina. Serie de informes de buenas prácticas del Departamento de Desarrollo Sostenible, Banco Interamericano de Desarrollo. Washington, D.C: Banco Interamericano de Desarrollo.

Mac Donald, J. (2004). Pobreza y precariedad del hábitat en ciudades de América Latina y el Caribe. Serie Manuales, 38. Santiago de Chile: División de Desarrollo Sostenible y Asentamientos Humanos, CEPAL, Naciones Unidas. 
Mitlin, D. \& Satterthwaite, D. (2004). Addressing deprivations in urban areas. En D. Mitlin $\&$ D. Satterthwaite (Eds.), Empowering squatter citizen. Local government, civil society and urban poverty reduction (pp. 245-277). London: Earthscan.

POLIS (2002). Aspectos económicos de experiencias de desenvolvimiento local. PÓLIS Estudos, Formação e Assessoria em Politicas Sociais, 40.

Programa de las Naciones Unidas para el Desarrollo (PNUD) (1997). Informe sobre desarrollo humano 1997. Nueva York: Ed. Mundi-prensa, España y Naciones Unidas.

Simioni, D. \& Szalachman, R. (2006, junio). Primera evaluación del programa regional de vivienda social y asentamientos humanos para América Latina y el Caribe. Informe regional. Documento presentado a la $15^{\text {a }}$ reunión de Ministros y Autoridades Máximas de la Vivienda y el Urbanismo de América Latina y El Caribe - $11^{\circ}$ Foro Iberoamericano de Ministros y Autoridades Máximas del Sector del Desarrollo Urbano y Vivienda, Montevideo, Uruguay.

Stallings, B. \& Weller, J. (2003). La generación de empleo en América Latina en los años noventa: base fundamental de la política social. En A. Puyana \& G. Farfán (Coords.), Desarrollo, equidad y ciudadanía. Las políticas sociales en América Latina (pp. 143-180). México, D.F.: FLACSO, Plaza y Valdés.

Tokman, V. E. (2004). Las dimensiones laborales de la transformación productiva con equidad. Serie Financiamiento del Desarrollo, 150. Santiago de Chile: Unidad de Estudios Especiales, Secretaría Ejecutiva, CEPAL, Naciones Unidas.

Vidler, E. (1999). City economic growth. Theme Paper $N^{o} 1$, Urban governance, partnership and poverty. Birmingham: International Development Department, School of Public Policy, University of Birmingham.

Wodon, Q.; Castro-Fernández, R.; Lee, K.; López-Acevedo, G.; Siaens, C.; Sobrado, C. \& Tre, J.P. (2001). Poverty in Latin America: trends 1986-1998 and determinants. Cuadernos de Economía, 114. 
